\title{
Research on the Application of BaChu Cultural Elements in Web Interface Design
}

\author{
Zheng Dandan ${ }^{1,}$, Zhang Ruizhi \\ ${ }^{1}$ School of Arts and Communication, China University of Geosciences, Wuhan, China \\ ${ }^{2}$ Bachu Art Culture Research Center, Yichang, China
}

\section{Email address:}

20207741@qq.com (Zheng Dandan)

${ }^{*}$ Corresponding author

\section{To cite this article:}

Zheng Dandan, Zhang Ruizhi. Research on the Application of BaChu Cultural Elements in Web Interface Design. Science Innovation. Vol. 7, No. 3, 2019, pp. 90-94. doi: 10.11648/j.si.20190703.12

Received: July 8, 2019; Accepted: August 3, 2019; Published: August 27, 2019

\begin{abstract}
The article highlights the importance of cultural elements in the relevant $\mathrm{Ba} \mathrm{Chu}$ web interface design so as to reflect $\mathrm{Ba} \mathrm{Chu}$ culture. It analyses the visual elements of texts, colors, graphic image, layouts dynamic effects and interaction theories, based on which to put forward the inner spirit and external performance of $\mathrm{Ba}$ Chu culture and expand the international vision of the traditional cultural elements in the application $\mathrm{Ba} \mathrm{Chu}$ culture in the web interface design and its development trend.
\end{abstract}

Keywords: Bachu Cultural Elements, Web Interface Design, Features

\section{巴楚文化元素在web界面设计中的应用研究}

\section{郑丹丹 ${ }^{*}$ ，张睿智 2}

1 中国地质大学艺术与传媒学院, 武汉, 中国

${ }^{2}$ 三峡大学巴楚艺术发展研究中心, 宜昌, 中国

\section{邮箱}

20207741@qq.com（郑丹丹）

摘要: 如何在web界面设计中体现巴楚文化元素, 并强调巴楚文化元素在相关巴楚web界面设计中的重要性。文章结合 web界面设计中文字、色彩、图形图像、布局、动态效果以及交互理念这六大视觉元素展开分析, 并在此基础上阐述 巴楚文化在web界面设计中的应用及未来的发展趋势, 提出巴楚文化元素内在精神外在表现并拓展传统文化元素国际 视野的内容。

关键词：巴楚文化元素, Web界面设计, 特色

\section{1. 引言}

“党的十八大报告中，提升文化软实力与保持经济健 康发展、提升人民生活水平等一起，被列为实现·建成小 康社会'目标的重要任务, 文化产业的经济支柱性产业地 位在这里再次被确认。”[1]现代人的生活, 在网站中汶览、
工作、学习已经是再寻常不过的事情, 但纵观国内大部分 网站亦缺乏设计美感、又或者是借鉴、挪用西方的审美元 素、混乱的信息架构以及不合理的视觉化编排给用户带来 了糟糕的视觉或情感体验。因此, 网站的设计与开发就显 得尤为重要, 成为企业发展、强国建设的重要组成部分。 
“视觉设计是网站内容、架构与交互模式的最终呈现, 是和用户进行交流的对话层, 它的好坏直接影响用户的使 用感受。”[2]如何让联网上的海量信息以最佳的视觉程序 快速准确地传达出去, 使汶览者能够通过自己的视觉经验 和心理联想认识理解这些视觉元素, 并能把设计者想要表 达的物质信息和精神信息良好的接受下来并将它传承下 去, 基于此, 本文从web界面设计中信息视觉的六个方面: 文字、图片、色彩、页面布局、动态效果以及交互理念来 分别展开讨论, 通过正反实例进行剖析和一系列探讨, 分 析未来web界面设计发展趋势与国内用户的审美取向。

\section{2. 巴楚文化元素与web界面设计}

“什么才是中国传统文化元素? 在张艺谋的创作中, 京剧的表现方式是中国元素; 在吴冠中的画中, 水墨意境 就是中国元素; 在梁思成的心中, 飞檐峭壁就是中国元 素......中国传统文化元素是中国文化的精髓, 并延续到我 们现代生活中来，更起到传承民族文化的作用......”[3]相 关巴楚文化的网站必将受到其传统文化因素的影响, 所形 成的意识形态也必将影响到它的网站界面设计。

\section{1. 何谓巴楚文化}

巴楚文化是巴文化与楚文化相互融合的结果, 这是巴 楚两地的物质、文化、风俗相互渗透而产生的一种新文化, 巴楚文化元素种类众多: 漆器、乐器、语言、社会风俗、 礼仪以及舞蹈等等, 各种各样的艺术形式都充分孕育着巴 楚文化的内涵。由于人类文化产的地域性、民族性的不同， 形成了与其他民族不一样的语言、道德、文化价值和审美 观念, 就形成了独特的巴楚地域文化。

\section{2. web的巴楚文化设计思想}

Web界面设计是将文字、图形图像、色彩、动画等这 些视觉元素合理地应用于网页界面, 并从视觉信息传达的 角度, 深入分析色彩、形态及其组合方式, 以期更有效率 的地达到信息传播的目的。网页界面具有强大的传播速度、 广泛的传播范围以及独特的交互化等优势, 巴楚文化元素 可以激发web界面中更多的交互动效，声音视频媒体的、 $3 \mathrm{D}$ 动态展示、虚拟场景的再现......, 这些都成为了web界 面设计师的灵感来源, 设计师经常通过对传统文化的研究 来解决网页界面设计风格的问题。

\section{3. 巴楚文化元素在相关巴楚web界面设计中是不可缺少 的因素}

巴楚文化元素是设计相关巴楚网页的设计师的灵感 来源, 除了这是展现web界面自身的文化性和个性以外, 也是让用户汶览网站时一眼就能感受到网站的特色巴楚文化。Web界面设计思维的起始点往往是展现网站自 身的文化特色, 最终用户在登录网页时能够区别于其他网 站并可以传达自身完整独特文化信息的web界面设计。

“不同的网站需要有不同的风格, 其风格要与主题内 容相统一。”[4]在web界面设计中, 不同文化背景下的网 页界面是可以通过设计的语言进行相互的沟通, 并可以在 各自的网页中体现各自的精神追求。如英国的web网站设 计, 就有英伦田园风、英伦校园风、另外日系风、韩系风、 欧美风格的网站特色都比较鲜明等等, 在色彩搭配上、排 版构图上带有各自文化特色的网页往往都是比较吸睛, 其 网站设计观点都是力图在传统与现代、西方与东方、民族 与地域之间通过网页界面设计将民族性与国际性的交融 表现出来。

\section{3. 巴楚元素在web界面中的运用}

“对于美感体验的需求，是人性的基本需求之一。”[5] 所有产品都具有两种最基本的价值属性: 实用价值和审美 价值。在web界面设计中毫无疑问同样适用, 事实上界面 设计的美感通常还使用户对web网站的实用价值的认知起 到禆益的作用, 随着中国互联网规模的迅速膨胀, 网站技 术进一步的发展和普及, 短短数年间已经发展成为了一门 新兴艺术门类, 早已改变传统中以技术为主导的局面, 但 相对于其他传统艺术设计专业来说, 网页界面设计更关注 的是艺术与技术的结合, 形式与内容的统一以及交互与情 感的诉求。界面设计大致可分为结构设计、交互设计和视 觉设计三大类, 视觉设计是呈现给用户最直观的部分, 也 是用户与电子产品直接互动的窗口, 此篇文章也主要围绕 网页的视觉设计这个层面展开论述。

网站的整体形象来自于网页中各视觉元素的特征组成, 以及各网页之间的结构组织关系和对汶览者所产生的视觉 心理影响。网页的视觉元素包括文字、图形图像、色彩、 布局、动态效果、交互理念六种, 下面分别就这六种元素 谈谈巴楚文化遗产如何在Web页面中更好的传承下来。

\section{1. 文字}

文字成为专门记录语言的特殊书写符号系统, 是人类 社会发展进入文明时代的一个重要标志, 也是人类进行思 想和情感交流的重要手段。“......不同风格的字体会产生 不同的视觉情感, 在选择字体时要保持文字的视觉感受与 表述内容的统一，从而创建出期望的风格和基调。”[6]少 数民族语言文字不仅是少数民族日常生产生活重要的交 际工具, 而且是民族文化的载体, 是民族情感的纽带, 也 是民族的基本标识之一。当前一些巴楚地区的门户网站未 体现应有的当地文化特色, 巴楚地域乃今天三峡地带, 这 里现实的存在着土家族文化、苗族文化、侗族文化、白族 文化、汉族文化等多个民族大杂居小聚居的格局形式, 这 些少数民族虽有各自民族语言却没有相应文字, 有些民族 虽有早期本民族文字，但由于使用范围比较小，有些已经 失传不再通用, 这种即将消失的或已然消失的语言文字我 们可以找到它并尝试在网页中使用它。例如土家族有自己 民族的语言, 却没有自己的文字, 属于汉藏语系藏缅语族, “毕兹卡”是土家族人的自称, 意为“本地人”; 称苗族“白卡”, 意为“邻居”; 称汉族“帕卡”，意为“客家”等等，为了再现 土家族语言而采用《土家语拼音方案》记音, 用汉字注音, 并用湘西汉语方言拼读注音。

另外, 从技术角度来说, Web界面设计上的文字传播 媒介是屏幕, 与传统的纸质显示有比较大的差异。这首先 
表现在, 屏幕文字是依靠像素来实现其清晰度, 不适合过 于细小的文字显示; 其次, 屏幕的亮度与光的漫反射作用, 以及屏幕的反光都会对用户的阅读产生影响; “在现代视 觉传达设计中, 字体及字号的合理选择, 科学的版面排布 方式以及合理的图文搭配能进一步发挥文字这一民族文 化符号的审美价值, 让市民在进行视觉欣赏的过程中收获 文化。”[7]因此, 语言文字也是一种非物质文化遗产, 也 会随时消失, 我们可以在设计中适时加入这些民族文字, 不但可以向广大读者展现民族文化, 更可以让这种民族文 字代代相传, 因为它集聚了中华民族先民的劳动智慧, 所 以笔者认为符合人视觉构造的文字编排与民族文字的传 承相结合就显得至关重要了。

\section{2. 图形图像}

图形图像与文字相比, 具有更形象、更直观、更强的吸 注性特点, 也是继文字之后最早引入到网络中的多媒体组成 元素。“现代产品中常常运用一定的传统图案来表达具有一 定叙事意义的设计思想。”[8]在Web页面设计中也是最容易 体现巴楚文化的视觉元素之一，例如巴楚民间信仰习俗主要 积淀在峡江地区的土家族民俗中, 廪君蛮的白虎图腾、楚人 的风图腾, 以及土著居民的龙图腾和后来汉民族带来的龙风 图腾文化在这里交融流传, 在这一地区, 似乎仅有龙凤呈祥 还不够, 另外再加上一只活力十足的白虎才更能表达本族人 民虔诚的崇拜之意, 尤其是在旅游相关网站界面设计中更应 该体现。例如: 三峡大坝旅游网站http://www.sxdaba.com中 几乎看不到任何关于巴楚地域的图形图像元素, 仅仅在介绍 三峡大坝景区一一屈原故里时, 界面中出现屈原雕像和一座 屈原祀建筑图像拼凑在一起, 剓归县屈原传说被列入第二批 国家级非物质文化遗产，具有浓厚浪漫主义气质的爱国主义 诗人屈原创作的《离骚》, 其作品中透露着楚人典型的多情 善感、想象力丰富的艺术世界, 可适当将屈原诗作作为界面 设计元素来体现巴楚文化特色。

相较于文字的识别率, 图片的视觉冲击力就大于文字 的 $85 \%$ 以上, 人们更容易被丰富多彩的图片所吸引。《设 计的法则》中谈到: “用图像优势效应来提高对关键信息 的认知和记忆。用图像和文字的结合来确保对关键信息的 强化, 已获得最佳效果。”[9]我们在设计时可以把巴楚地 区这些远古神话传说和图腾符号等元素提炼出来, 配合上 与之相适应的界面风格, 可使整个网页界面的设计更富有 文化底蒀。例如现在比较流行的扁平化、拟物化界面风格 等, 或者也有传统的水彩、版画等各种绘画风格以及电子 设备处理、3D技术实现图形等技术含量较高的界面风格可 供选择, “与书籍等传统媒体不同的是, 汶览网页的过程 是连续的、可选择的, 用户对信息的接受是非单线性的。 信息的隐藏、展开以及跳转在很大程度上依赖于图形的引 导。”[10]总之, 传统文化与现代科技手段的融合自然是相 得益彰、一气呵成, 并不矫揉造作。

\section{3. 色彩}

色彩的感觉是一般美感中最大众化的形式。[11]色彩 也是设计师重要的表现语言与手段之一, 不同的色彩能带 给人们不同的审美感受, 不同人对色彩的审美感觉也是各
不相同, 针对巴楚地域网页界面设计则应该利用色彩体现 当地民族性、地域性特点来。例如楚式漆器髹饰技艺作为 楚文化的支柱, 技艺古老充满神秘性而成为全国非物质文 化遗产的保护对象, 自古就有楚人尚赤的说法, “赤” 红色, 而楚地漆器的红并不似中国传统年画中的大红, 也 不似西方野兽派画中纯色的红, 它是典雅的、高贵的、浪 漫的暗红。在黑色背景中这种红色更有利的凸显了出来, 在此基础上辅以黄、绿、蓝、白等色, 这种传统久远的历 史色彩, 可能源于“禹作为祭器, 黑漆其外, 而朱画其内” 的传统 ( 《韩非子・十过篇》) 。如战国时期楚国的重要 乐器一一虎座鸟架鼓, 距今已有二千二百多年的历史, 其 上鬆黑漆为地, 以红、黄、金、蓝等色绘出虎斑纹和风的 羽毛, 象征着楚人崇鸣风、向往和平安详的意识和敢于征 服猛兽、不畏强暴的精神。

“在民族色彩喜好影响下, 每种民族元素本身也有自 己的色彩...审美有时代性, 不同时代的审美各有不同, 少 数民族元素虽然具有民族化、乡土化等审美特征，但是同 时民族图案也大多是民族生活实践的高度抽象, 具有抽象 性特点。”[12]例如http://bcys.ctgu.edu.cn/三峡大学-巴楚艺 术发展研究中心网页, 设计者主观的设置色彩, 通篇网页 上呈现的是以赫石色为主色调, 无论是背景色还是标题处 图形都被形象条理化, 这是一个典型展现色彩理想性的网 页; 而色彩的象征性, 比如楚人远祖, 乃天上的日神炎帝, 是地面的火神祝融, 日与火皆为红色, 在楚人看来, 红色 能带给人类光明和温暖, 象征着万物如火如茶, 生活富裕 美满等寓意; 而和谐性则是一种色彩搭配的对比调和方法, 简单来说画面中出现补色关系的对比色, 如红与绿、黄与 紫等让人感到很刺眼, 可以通过改变色彩面积或者降低色 彩纯度或明度, 或者加入黑白灰中性色等方法进行调和使 网页界面色彩趋于和谐,如www.sxdaba.com三峡大坝网站 主页的背景色是蓝色调的大坝, 而页面底部摆上一排黄色 小花, 仅从色相上看对比就有些不和谐, 另外没有控制好 网页的视觉层次等问题。

色彩有它的民族性、地域性以及时代性，巴楚地区富 有特殊含义的民族色彩情感也应该纳入到我们设计者思 考的范围, 应该采用何种色彩也应要与网页内容相符合, 内容决定形式。总之, 巴楚地区网页界面色彩的使用是要 体现其理想性、象征性以及和谐性。

\section{4. 页面布局}

布局设计是根据网站的内容和建站目的, 运用版面设 计原理, 将网页上的视觉元素进行合理配置与表现的过程。 [13]布局设计的过程不是简单地摆放各种元素, 而是为实 现页面信息传递的清晰化、合理化、人性化，同时考虑视 觉形态的美化。有了前面论述到的巴楚文化遗产元素, 接 下来就是设计好页面秩序, 使它具有更高的视觉度。建立 页面秩序需要考虑的因素比较多, 笔者总结产生影响的主 要有以下四个方面。

1) 图版率: 图版率即版面率, “版面率是网页页面中 的文字、图形、图像、动画、视频等的占有率, 它影响着 页面信息传递的效率, 也决定了整个页面的视觉传达效 果。”[14]大版面率符合目前的流行设计风格, 即在大背景 
下加上少量的文字设计。例如2017年www.apple.com苹果 官方网站首页，艺术家们用Apple产品来创作年画的页面 极好的演绎了如何将传统文化融入现代设计之中。

2) 网格的约束率：使页面布局显得更加严谨、稳重 和理性。例如www.cn3x.com三峡宜昌新闻网站首页, 整 个页面大体上看有条若影若现的网格线进行了信息分区, 但由于文字排列较散, 整个页面给人在视觉汶览上不够清 晰, 明了和整体。相比较www.sohu.com搜狐门户网站, 文字信息量自是不言而喻, 但作为用户汶览下来发现通过 文字字体大小、粗细对标题与内容进行很好的规划区分, 再加上不同深浅、颜色的网格线使密集的信息量更加条理 化、规整化。

3）跳跃率: 通常文字跳跃率大的页面会显得活跃, 反之会显得平静沉着。例如www.morehazards.com一个音 乐类网站就使用了较高的文字跳跃率, 整个页面显得比较 活跃, 所以跳跃率的高低会与网站的类型以及页面所要传 达的内容与功能相吻合。

4) 布局样式: 通常布局样式有水平分割、垂直分割、 网格分割、混合分割和自由分割等。例如www.1905.com 电影网是一个介绍最新电影资讯的门户网站, 网站简洁利 落的设计风格, 海报图片与文字介绍的有机搭配使整个网 站看起来处处有“亮点”, 当然这也和网站自身信息内容的 “吸睛度”有密不可分的关系, 不可否认的是页面很好的将 水平分割与垂直分割相结合的结构, 对信息区域进行合理 划分，页面布局丰富、灵活、实用，这无疑为巴楚地域的 网页页面设计提供了很好的借鉴作用。

布局是运用科学合理的版面设计原理来进行界面的 设计, 在巴楚地区网页界面中无疑是设计师必须掌握好 的一项基本技能, “网页布局是网页精彩与否的重要因素 之一, 网页布局关系着内容的编排和页面的排版, 它直 接影响着网页信息的浏览及体现出网页的美感。”[15]这 是由设计师自身专业素质所决定的, 好的布局设计会让 用户拥有一个良好的使用体验, 同时视觉上也会让人赏 心悦目。

\section{5. 动态效果}

动态元素是Web界面设计中展示信息的另一个独特 的方式, 毫无疑问它所承载的信息量也远高于一页纸的内 容。“......界面设计并非简单的艺术设计，平面设计画面 以静态、视觉焦点比较直观、图像完整性强为特点, 而界 面设计拥有诸多动态元素, 视觉感受上活泼、灵活, 还能 引导用户进行互动性操作。”[16]早期GIF动画到风靡一时 的flash动画, 再到Java script动效, 而现在流行的Html5动 画、CSS动画等动态展示技术不断的丰富着我们的页面设 计, 它带给我们的更多是一种丰富的、灵活性的和趣味性 的视觉体验, 更确切地说是为交互问题而设计, 以此来吸 引更多浏览着的关注。目前在一些传统文化的网页中还未 见到这种交互式的动态展示, 这无疑是一种有意义的尝试, 也是一种打破一般传统视频动态的新方式。例如《华阳国 志・巴志》载: “巴师勇锐, 歌舞以凌, 殷人前徒倒戈, 故 世称之, 武王伐纣, 前歌后舞也。”有了数千年的历史, 这种土家族特有的丧葬仪式与原始宗教有着密切关系, 舞
蹈唱腔中包含着对图腾崇拜和祖先崇拜所逐渐形成的民 间宗教信仰特征。可以尝试把这一仪式过程提取典型片段 制作成Java script动效, 或者是Html5动画形式来页面中展 示等。

随着虚拟现实技术中三维展示技术的不断发展, 越 来越多的行业开始应用这种更加直观真实的方式来对自 己的公司以及产品进行宣传。[17]当然, 在这些巴楚文化 中还有很多是可以采取这种形式展示出来的, 例如荆楚 主要民间音乐——宜昌丝竹, 特色是曲调细缄, 典雅清 新、旋律华丽、板式规范, 此小调又与本地民歌交融汇 合成一种独特的民族风格; 重庆市铜梁县以龙为主要道 具的汉族民俗舞蹈一一龙舞, 兴起于明, 鼎盛于清。它 既是舞蹈, 又是体操与杂技, 舞时烟花烧龙、舞时音乐 独特、舞蹈者服饰简朴大方等特色无疑都可以“载入”到 巴楚地域 $\mathrm{Web}$ 页面之中, 声音和动作的动态效果展示一 定可以丰富、创新和向世界各族人们展示巴楚文化遗产 的风采。

\section{6. 交互理念}

“用户上网的目的、需求才是关键点。能吸引用户的 网页, 都能够将交互理念贯彻与网页界面设计之始 终。”[18]web产品测试是贯穿于整个设计的始终, 要求界 面设计师始终站在用户的角度去思考交互流程是否好用, 例如模拟用户操作使用的流程、不断更新迭代自定义功能 设计、在web结构上是否条理清楚、可维护性是否方便操 作等等, 这些都影响用户体验是否良好, 能否将用户发展 成为网站的忠实用户。

“网站设计=网站整体策划+网站界面设计+程序编程 设计+功能测试+后期维护” [19]本文所要研究的是如何设 计巴楚文化网站形象的界面风格, 首先在设计之前就必须 梳理出网站所要传达的内容框架和逻辑架构图, 这样才能 满足用户的功能需求和审美需求, 到逐渐关注用户的精神 需求。

\section{4. 结论}

“在全球新一轮科技革命和产业变革中, 互联网与各 领域的融合发展具有广阔前景和无限潜力, 已成为不可阻 挡的时代潮流, 正对各国和地区经济发展产生着战略性和 全局性的影响。”[20]巴楚文化是传统文化其中之一，成为 政府、媒体、公众关注的焦点之一, 如何让它更好的传承 下来就是要让传统文化和现代技术相结合, 能够用现代语 言把传统文化表达出来就是一种创新, 这是一项文化创意 产业, 是以技术为核心载体的现代产业, 它的市场只能是 全球性的。“重塑传统文化的途径绝不是“复古”, 而是要培 植或重塑文化传统的“生命力’。”[21]“中国元素不是表面 的装饰, 它依靠的是好的创意带来的内在逻辑, 或者说, 它应转换成适合时代的图像载体。”[22]在现代设计理念的 助推下, 把它运用于Web界面设计当中, 借助于互联网的 优势, 让更多的人去了解巴楚文化, 更好的传承巴楚地域 的文化。 


\section{基金项目}

湖北省高校人文社科重点研究基地巴楚艺术发展研 究中心开放基金项目（2016178003）。

\section{参考文献}

[1] 王莉, 《地域特色在旅游产品包装设计中的应用》, 艺术 百家, 2014。

[2] 薛艳敏, 戴毓: 《网页设计元素对PAD情感体验的影响研 究》, 装饰, 2018.02月。

[3] 张赛娟, 《中国传统文化元素在动画造型设计中的运用》, 艺术百家，2010.07期。

[4] 那日图, 《少数民族文化艺术元素在网页设计中的运用》, 美苑，2014.06期。

[5] 贾京鹏: 《界面设计》, 中国青年出版社, 前言。

[6] 秦岁明, 何梦楠, 《扁平化风格在网页界面设计中的应用》, 包装工程，2015.03月。

[7] 陈辉, 《民族文化符号在视觉传达设计中的运用分析》, 南京艺术学院学报（美术与设计），2016.06期。

[8] 梁黎, 《中国传统图案在手机UI界面中的设计应用研究》, 艺术评论，2016.03月。

[9] 威廉·立德威尔.《设计的法则》[M]. 李婵, 译.沈阳: 2010。

[10] 遆佳, 李雯, 《基于视觉信息传达的网页界面设计研究》, 包装工程，2017.01月第2期。

[11] 中央编译局.《马克思恩格斯全集》 [M]. 北京: 人民出版社, 1963。

[12] 王峡, 《传统民族元素在现代平面设计中的应用思考》, 贵州民族研究，2016.11期。

[13] 隋涌, 《视觉设计》, 清华大学出版社, 2015年3月, 第29 页。
[14] 杨西慧, 刘西莉, 《网页设计的视觉要素与编排创意》, 装饰，2005.04月，第144期。

[15] 丁海燕, 《网页设计布局方法的探讨》, 云南大学学报 (自 然科学版)，2013.35（S1）期。

[16] 刑蓬华, 《移动界面的情感化交互设计》, 美术观察, 2017.06 月。

[17] 陈艳红, 古丽米拉·克孜尔别克, 谢卫国, 吕永杰, 《web 页面三维动态展示技术研究与应用》, 现代电子技术, 2018.10.15，第20期。

[18] 熊璐, 《以交互理念为核心的网页界面设计课程教学实践 探索》，装饰，2019.总第313期。

[19] 史金玉, 《基于用户体验的网站界面设计研究》，艺术工 作，2017.02期。

[20] 王友奎, 周亮, 张少彤, 汪敏: 《“互联网+”战略下中国政 府网站发展的新要求与新趋势》, 电子政务, 2016.02期。

[21] 吴剑锋, 《从“符号”到“意象”一传统文化在中国当代设 计艺术中的诗性表达》, 浙江社会科学, 2016.11期。

[22] 汪瑞, 《中国元素未必等于中国设计》, 装饰, 2010.01, 第201期。

\section{作者简介}

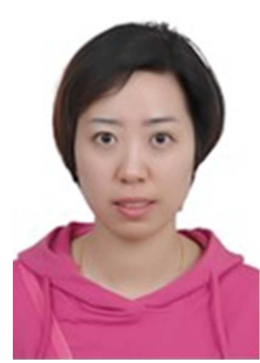

郑丹丹（1980—），女，湖北鄂州 人, 中国地质大学艺术与传媒学院在 读博士生、讲师。 